\title{
Conformational changes in wheat gluten after using Ag-nanoparticles
}

\author{
Agnieszka Nawrocka \\ Institute of Agrophysics, Polish Academy of Sciences, Doświadczalna 4, 20-290 Lublin, Poland
}

Received April 9, 2013; accepted June 2, 2013

A b s t $r$ a c t. Silver nanoparticles have antimicrobial properties since they can be regarded as an efficient protector against pathogenic microorganisms. Fourier transform infrared spectroscopy was used to examine conformational changes in the secondary structure of wheat gluten washed out from grain treated with an aqueous solution of silver nanoparticles stabilized by trisodium citrate. Silver nanoparticles were used as a protective layer on the grain surface against bacterial and fungal infections (antimicrobial agent). Analysis of the amide I band revealed significant changes in the secondary structure after using silver nanoparticles. An increase in the $\beta$-sheet content (from 36.2 to $39.2 \%$ ) was observed at the expense of the $\alpha$-helix and $\beta$-turn content. To find factors causing these changes, the wheat grains were treated by an aqueous solution of trisodium citrate and water. The results obtained indicate that the changes in the gluten structure were connected mainly with the trisodium citrate action due to presence of a small number of free molecules of the stabilizer in the solution of silver nanoparticles. Additionally, the conformational changes in gluten pointed out that gluten flexibility increased (decrease in the $\alpha \mathrm{H} / \beta \mathrm{S}$ ratio from 1.40 for the control sample to 1.26 for the silver nanoparticle-treated samples) as well as the solubility of gluten decreased (decrease in the $\beta$-turn content from 13.1 to $11.4 \%$ ).

$\mathrm{K}$ e y w o r d s: silver nanoparticles, trisodium citrate, wheat gluten, FTIR spectroscopy, secondary structure

\section{INTRODUCTION}

Annually, over two billion tons of plant products are produced in the world. Crop storage/transport carried out in proper conditions is crucial for avoiding heavy losses in yield and providing food with a large number of nutrients and of the highest quality (Jayas and White, 2003). Postharvest losses are mainly due to bacteria and fungi infections. The microorganisms contaminate the stored/transported crops with metabolites eg mycotoxins, and significantly diminish the quality of plant products. Although there are

Corresponding author e-mail: a.nawrocka@ipan.lublin.pl adequate regulations concerning grain storage, food is still contaminated by pathogens and their metabolites. For this reason, an efficient protector is needed to prevent the grain and other food products ( $\mathrm{g}$ fruits, vegetables) from development of pathogenic microorganisms. Silver nanoparticles (AgNPs) seem to be a good candidate for a protective substance due to their antimicrobial properties in a broad spectrum.

Silver has been used for centuries in the form of metallic silver, silver nitrate, and silver sulfadiazine for treatment of burns, chronic wounds, and bacterial infections. However, introduction of antibiotics declined significantly the use of the silver compounds (Rai et al., 2009). Recently, silver compounds have come up due to increasing microorganism resistance to antibiotics. Especially silver nanoparticles are widely used because they have proved to inactivate bacteria, fungi, viruses, and algae (Marambio-Jones and Hoek, 2010). For this reason, NPs have been applied in medicine, water and air purification, food production, cosmetics, clothing, and numerous household products. These broad applications, however, increase human exposure and thus the potential risk related to their short- and long-term toxicity. Some authors have claimed the AgNPs possess low or zero toxicity to human cells (Barrena et al., 2009; Duran et al., 2007). However, a large number of in vitro studies show that silver NPs are toxic to mammalian cells derived from skin, liver, lung, and brain (Ahamed et al., 2010). Generally, the toxicity of AgNPs depends on their size, shape, chemical composition, and surface modification (BystrzejewskaPiotrowska et al., 2009).

Although the mechanism of the action of Ag nanoparticles on cells (fungal, bacterial, and human) is not recognized precisely, different silver technologies have been approved by the Food and Drug Administration (FDA) and the European Food Safety Authority (EFSA). Applications 
of silver nanotechnologies in the food industry include coatings of machines and storage containers, the use of nanosieves to filter out bacteria, biodegradable starch/clay films implemented by AgNPs used in packaging materials, and engineered nanoparticles utilized as food additives or supplements in functionalized foods. Nanoparticles are also used as the so-called nanosensors in packaging materials to indicate environmental changes (eg temperature or moisture), degradation products of food commodities, and contamination by pathogen microorganisms (Bouwmeester et al., 2009). AgNPs can also be employed as absorbent pads in food packaging to absorb moisture and fluids exuded from meat and fish, keeping the products looking fresh and creating an aesthetically attractive packaging (Fernandez et al., 2009).

Silver nanoparticles are used in paints covering silos or containers, but they have not yet been applied to protect directly stored/transported grain against fungal and bacterial infections. The aim of these studies was to determine if and how silver nanoparticles stabilized by trisodium citrate influence the secondary structure of wheat gluten. Changes in the protein structure may affect the quality of wheat flour and bread-making process. Additionally, the influence of water and an aqueous solution of trisodium citrate on the gluten secondary structure was characterized.

\section{MATERIALS AND METHODS}

The study was carried out on winter wheat (Triticum aestivum L.). The wheat grain was obtained from the Strzelce Plant Breeding Station, Poland.

Silver nitrate $\left(\mathrm{AgNO}_{3}\right)$ and trisodium citrate (TC) were purchased from Sigma-Aldrich and used as received. Double distilled water was used.

Citrate-reduced and stabilized silver colloid was prepared according to a modified procedure reported by Lee and Meisel (1982). Briefly, $100 \mathrm{ml}$ of $1 \mathrm{mM} \mathrm{AgNO}_{3}$ was brought to boiling. A solution of $1 \%$ trisodium citrate $(2 \mathrm{ml})$ was added immediately under vigorous stirring. The solution was kept boiling and stirred simultaneously for $c a$. 10-15 min. A change of the solution colour to yellow was a sign of formation of spherical AgNPs. The absorption spectrum of the AgNPs solution was recorded on a Cary 300/Biomelt spectrophotometer (Varian Pty., USA) to confirm formation of nanoparticles. The absorption spectrum showed a surface plasmon resonance (SPR) absorption band with a maximum of $413 \mathrm{~nm}$ indicating the presence of spherical AgNPs with a diameter of $c a$. 20-30 nm.

This kind of nanoparticles was chosen due to their high antimicrobial activity (Guzman et al., 2009). Additionally, trisodium citrate (E331), used as a reducer and stabilizer of nanoparticles, is commonly used as a buffer, chelate agent, nutrient additive, and emulsifier in the food industry.

The samples of wheat grain $(100 \mathrm{~g})$ were stirred with $30 \mathrm{ml}$ of aqueous solution of AgNPs stabilized by trisodium citrate $\left(\mathrm{c}_{\mathrm{AgNPs}}=1 \mathrm{mM}\right)$, the aqueous solution of TC $\left(\mathrm{c}_{\mathrm{TC}}=1.4 \mathrm{mM}\right.$, and water. The concentration of TC was identical with the concentration of the compound used in the AgNPs preparation. Stirring was applied three times: 10 (T10), 20 (T20), and $30 \mathrm{~min}$ (T30). The control sample (T0) was not treated with any compound. After stirring, the wheat samples were air-dried for $24 \mathrm{~h}$ at a temperature of $22^{\circ} \mathrm{C}$ and milled.

Gluten was washed by using a Glutomatic 2200 washer (Perten Instruments, USA) according to Approved Method 38-12 (AACC, 2000). After washing, gluten samples were freeze-dried, milled, and used in the FTIR measurements.

Fourier-transform infrared (FTIR) spectra were recorded with a Nicolet 6700 FTIR spectrometer (Thermo Electron Corporation, USA) equipped with a diamond attenuated total reflectance attachment. The IR spectra were recorded between 4000 and $600 \mathrm{~cm}^{-1}$ at $4 \mathrm{~cm}^{-1}$ intervals. Each spectrum resulted from 128 scans to obtain an optimal signal-to-noise ratio. Each spectrum of gluten powder was divided by the background spectrum and converted to an absorbance spectrum. Additionally, the spectra were manually corrected with a linear baseline at four points (approximately $4000,3750,3600$, and $1750 \mathrm{~cm}^{-1}$ ) in the OMNIC software (version 8.2, Thermo Fisher Scientific, Inc., USA).

Structural analysis of the amide I $\left(1570-1720 \mathrm{~cm}^{-1}\right)$ and III bands (1200-1340 $\mathrm{cm}^{-1}$ ) was conducted using ORIGIN (version 9.0 PRO, OriginLab Corporation, USA). The analysis included baseline correction, derivation, curve fitting, and area calculation of the amide I band. Second derivative spectra of the studied bands were obtained using a ninepoint, two-degree polynomial function. The gluten amide bands of all the samples, including the control sample, wheat samples treated with the solution of silver nanoparticles, trisodium citrate solution, and water, were deconvoluted by the curves centred at the same wavenumber. In order to calculate the percentage contribution of the different types of conformation to the area of all components, bands assigned to a given conformation were summed and divided by the total area of the amide I band. The $\alpha$-helix-to- $\beta$-sheet ratio $(\alpha \mathrm{H} / \beta \mathrm{S})$ was calculated to indicate the molecular flexibility of the studied gluten samples.

The statistical analysis was carried out with STATISTICA (version 10, StatSoft Inc., USA). Univariate analysis of variance (ANOVA) was used to detect significant differences in the percentage distribution of $\alpha$-helix, $\beta$-sheet, $\beta$-turns, and the $\alpha$-helix-to- $\beta$-sheet ratio in the gluten samples versus the stirring time (T0, T10, T20, T30). p-values below 0.05 were considered significant.

\section{RESULTS AND DISCUSSION}

The peptide groups in proteins give nine characteristic vibrational bands (amides A, B, and I-VII) in the midinfrared region. Generally, only two of the bands, amide I $\left(1600-1700 \mathrm{~cm}^{-1}\right)$ and amide II $\left(1500-1600 \mathrm{~cm}^{-1}\right)$ are used to characterize the secondary structure of proteins. Sometimes also the amide III band $\left(1200-1350 \mathrm{~cm}^{-1}\right)$ is applied to 
determine the protein structure due to lack of water $(-\mathrm{OH})$ vibrations in this region. The IR spectrum of powder gluten washed from the wheat grain exhibited amide I, II, and III bands centred at 1648,1519 , and $1235 \mathrm{~cm}^{-1}$, respectively (Nawrocka and Cieśla, 2013). Location of the amide I band between 1640 and $1650 \mathrm{~cm}^{-1}$ indicated that gluten protein contained both $\alpha$-helical and $\beta$-sheet structures, since $\alpha$-helical proteins had an amide I band centred between 1650 and $1658 \mathrm{~cm}^{-1}$ while $\beta$-structural proteins were characterized by an amide I band situated between 1620 and $1640 \mathrm{~cm}^{-1}$ (Surewicz et al., 1993).

The secondary structure of the gluten samples was determined by analysis of the amide I band. Second-derivative transformation of the band showed three minima located approximately at 1621,1653 , and $1680 \mathrm{~cm}^{-1}$ (Fig. 1b). The deconvolution of the amide I band was made on the basis of the second derivative minima. The deconvoluted amide I band of washed out gluten (panel a) and its second derivative (panel b) are presented in Fig. 1. The particular components were connected with the following conformations: $1621 \mathrm{~cm}^{-1}$ - $\beta$-sheets, $1653 \mathrm{~cm}^{-1}-\alpha$-helix, and $1680 \mathrm{~cm}^{-1}-\beta$-turns (Carbonaro and Nucara, 2010). Powder gluten washed from the wheat grain (T0) contained $50.7 \%( \pm 0.6 \%)$ of $\alpha$-helices, $36.2 \%( \pm 0.6 \%)$ of $\beta$-sheets, and $13.1 \%( \pm 0.3 \%)$ of $\beta$-turns, while commercially produced wheat gluten included $34.5 \%$ of $\alpha$-helices, $48.8 \%$ of $\beta$-sheets, and $17.3 \%$ of $\beta$-turns (Liao

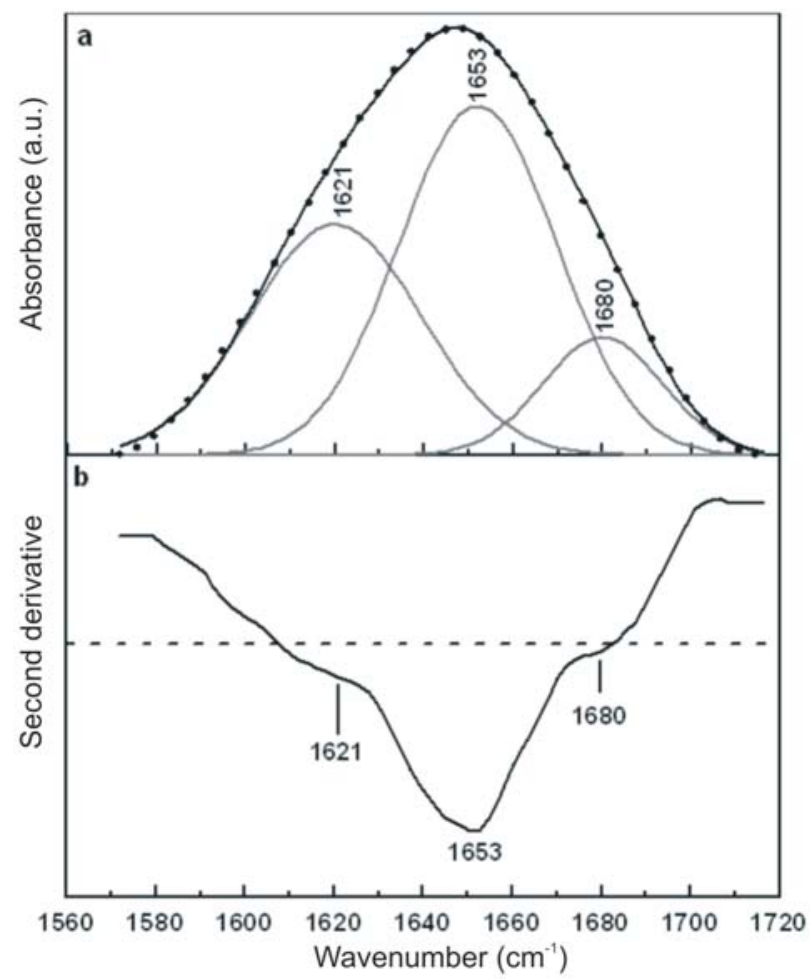

Fig. 1. Deconvoluted infrared amide I band of powder gluten (T0) (a) and its second derivative (b). The dashed line is placed at zero point. et al., 2010). The $\alpha$-helix-to- $\beta$-sheet ratio $(\alpha \mathrm{H} / \beta \mathrm{S})$ was equal to $1.40( \pm 0.04)$. IR spectroscopy was used by Lee $e t a l$. (1990) to determine the secondary structure composition of native gluten in an aqueous solution. The gluten contained $31 \%$ of $\alpha$-helices, $28 \%$ of $\beta$-sheets, $27 \%$ of $\beta$-turns, and $17 \%$ of random coils. Gluten washed from the wheat grain was rich in $\alpha$-helical structures (maximum of the amide I band located at $1648 \mathrm{~cm}^{-1}$, and $\alpha \mathrm{H} / \beta \mathrm{S}$ bigger than 1) and had fewer $\beta$-sheets and $\beta$-turns in comparison with commercial gluten as well as native gluten. Additionally, the studied gluten contained zero random coils.

Although the amide III band does not correspond mainly to one mode of vibration like amide I band ( $80 \%$ of $\mathrm{C}=\mathrm{O}$ stretching), it is used to estimate the secondary structure in proteins. The amide III region corresponds to $\mathrm{N}-\mathrm{H}$ in-plane bending (60\%) coupled with C-N stretching (40\%). The signal is weaker in the band but water vibrations do not interfere with the protein spectrum as in amide I. To confirm that the gluten structure contained three structural components ( $\alpha$-helix, $\beta$-sheet, and $\beta$-turns), the second derivative of the spectrum of the amide III band $\left(1200-1340 \mathrm{~cm}^{-1}\right)$ was calculated. The second derivative, presented in Fig. 2b, showed three minima located at 1234,1275 , and $1308 \mathrm{~cm}^{-1}$. According to Cai and Singh (1990), band assignments were established for the: $\alpha$-helix, $\beta$-sheet, and $\beta$-turns at: 1308 , $1234,1275 \mathrm{~cm}^{-1}$, respectively.

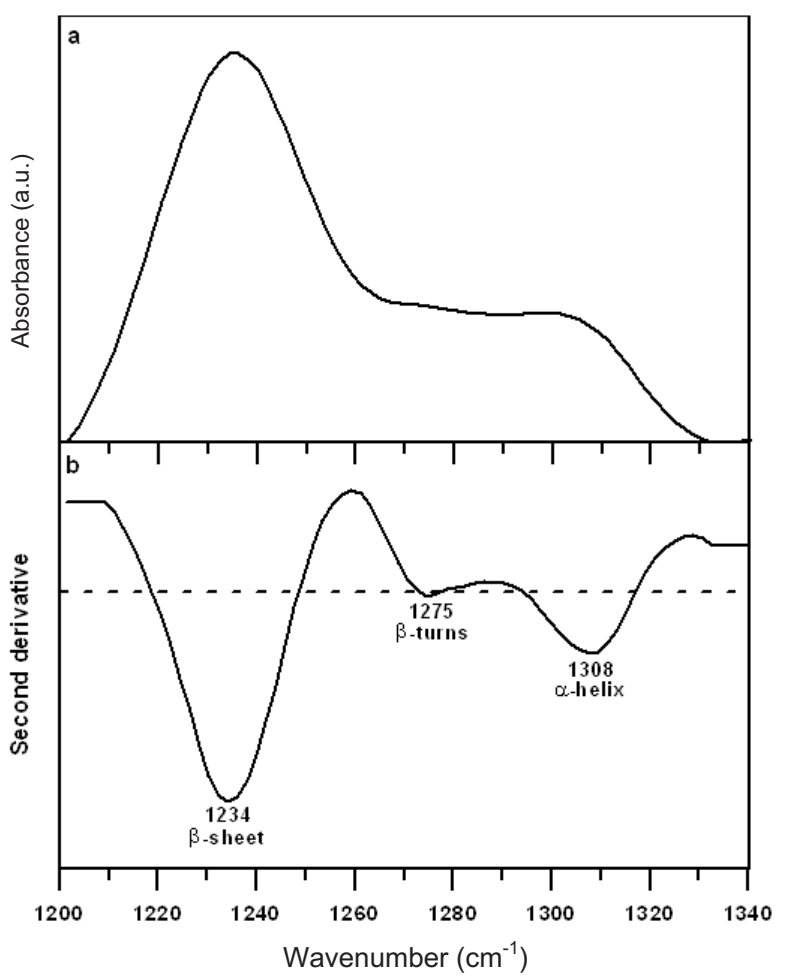

Fig. 2. FTIR spectrum (a) and second derivative (b) of the amide III band of powder gluten (T0) washed from wheat grain. The dashed line is placed at zero point. 


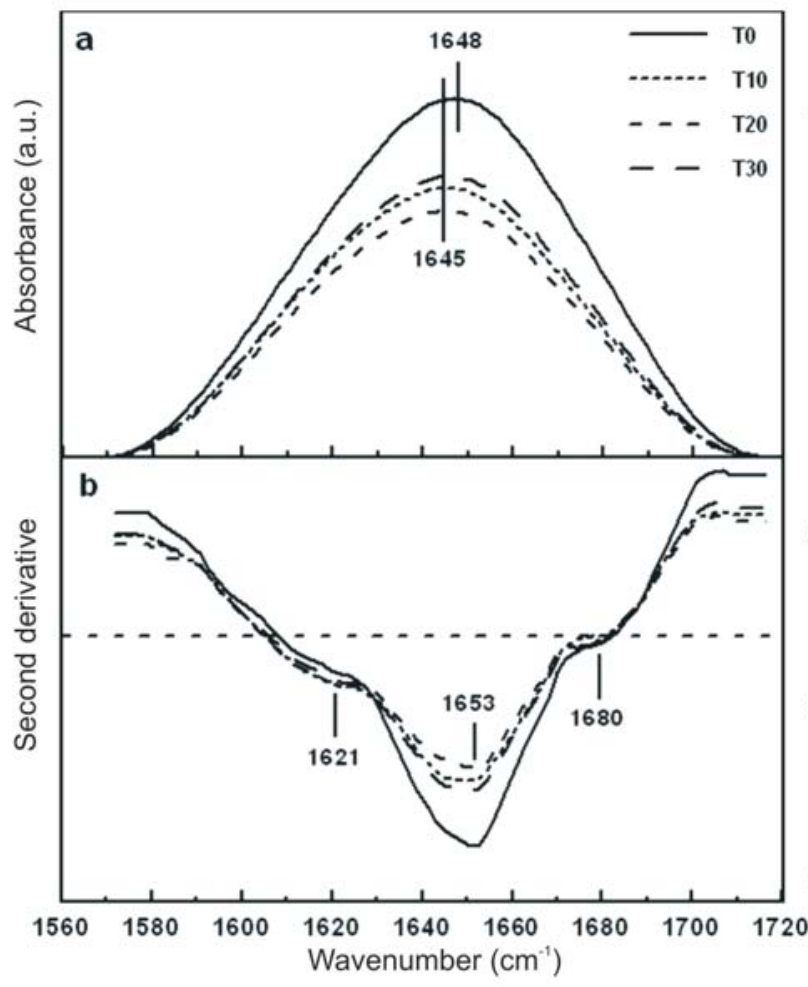

Fig. 3. Infrared spectra (a) and second derivatives (b) in the amide I region of untreated gluten (T0) and AgNP-treated gluten (T10, T20, T30). The dashed line is placed at zero point.
Infrared spectra of gluten samples treated with silver nanoparticles stabilized by trisodium citrate and their second derivatives are shown in Fig. 3. Analysis of the amide I band of gluten showed that using silver nanoparticles shifted slightly the band maximum to shorter wavenumbers (from $1648 \mathrm{~cm}^{-1}$ for T0 to $1645 \mathrm{~cm}^{-1}$ for T10, T20, and T30). Simultaneously, a decrease in the intensity of the band was observed. Second derivatives of the spectra were calculated. Similar to the control sample, second derivative spectra depicted three minima at 1621,1653 , and $1680 \mathrm{~cm}^{-1}$. The structural components connected with the $\alpha$-helix, $\beta$-sheet, and $\beta$-turns were assigned at 1621,1653 , and $1680 \mathrm{~cm}^{-1}$, respectively. The percentage contribution of the structural components and $\alpha$-helix-to- $\beta$-sheet ratio $(\alpha \mathrm{H} / \beta \mathrm{S})$ changed after using the AgNPs. The AgNP-treated gluten samples (T10, T20 and T30) contained 49.3\% $( \pm 0.4 \%)$ of $\alpha$-helices, $39.2 \%( \pm 0.6 \%)$ of $\beta$-sheets and $11.4 \%( \pm 0.2 \%)$ of $\beta$-turns on average. The $\alpha \mathrm{H} / \beta \mathrm{S}$ ratio decreased from 1.40 for the control sample to $1.26( \pm 0.03)$ for the AgNP-treated samples. Significant correlations between the structure distribution (for all structural components) and the stirring time were observed $(\mathrm{p}<0.0001)$ and are shown in Table 1.

Using colloidal AgNPs as a protective layer caused changes in the secondary structure of gluten. As seen in Table 1, $\beta$-sheet structures increased at the expense of $\alpha$-helical and $\beta$-turn structures after covering wheat samples with AgNPs. Furthermore, the value of the $\alpha \mathrm{H} / \beta \mathrm{S}$ ratio decreased to 1.26 , demonstrating that the nanoparticles modified wheat gluten

T a b l e 1. Structure distribution (\%) of gluten samples treated with an aqueous solution of silver nanoparticles stabilized by sodium citrate, aqueous solution of trisodium citrate, and water

\begin{tabular}{|c|c|c|c|c|c|}
\hline \multirow{2}{*}{$\begin{array}{l}\text { Secondary } \\
\text { structure }\end{array}$} & \multicolumn{4}{|c|}{ Structure distribution (\%) } & \multirow{2}{*}{$\mathrm{p}$} \\
\hline & T0 & $\mathrm{T} 10$ & $\mathrm{~T} 20$ & T30 & \\
\hline \multicolumn{6}{|c|}{ AgNPs stabilized by trisodium citrate } \\
\hline$\alpha H$ & $50.7(0.6)$ & $49.1(0.6)$ & $49.0(0.6)$ & $49.8(0.6)$ & \multirow{4}{*}{$<0.0001$} \\
\hline$\beta S$ & $36.2(0.6)$ & $39.5(0.6)$ & $39.6(0.6)$ & $38.5(0.7)$ & \\
\hline$\beta \mathrm{T}$ & $13.1(0.3)$ & $11.3(0.2)$ & $11.3(0.2)$ & $11.7(0.3)$ & \\
\hline$\alpha \mathrm{H} / \beta \mathrm{S}$ & $1.40(0.04)$ & $1.24(0.04)$ & $1.24(0.03)$ & $1.29(0.04)$ & \\
\hline \multicolumn{6}{|c|}{ Trisodium citrate } \\
\hline$\alpha \mathrm{H}$ & $50.7(0.6)$ & $48.3(1.0)$ & $47.6(1.1)$ & $48.4(0.7)$ & \multirow{2}{*}{$<0.0001$} \\
\hline$\beta S$ & $36.2(0.6)$ & $38.2(1.2)$ & $38.9(1.0)$ & $38.9(0.7)$ & \\
\hline$\beta \mathrm{T}$ & $13.1(0.3)$ & $13.5(0.4)$ & $13.5(0.4)$ & $12.7(0.4)$ & 0.0006 \\
\hline$\beta \mathrm{H} / \mathrm{bS}$ & $1.40(0.04)$ & $1.26(0.06)$ & $1.23(0.06)$ & $1.24(0.04)$ & $<0.0001$ \\
\hline \multicolumn{6}{|c|}{ Water } \\
\hline$\alpha \mathrm{H}$ & $50.7(0.6)$ & $48.5(0.4)$ & $47.5(0.8)$ & $48.1(0.4)$ & \multirow{4}{*}{$<0.0001$} \\
\hline$\beta S$ & $36.2(0.6)$ & $39.5(0.3)$ & $40.0(1.0)$ & $39.3(0.3)$ & \\
\hline$\beta \mathrm{T}$ & $13.1(0.3)$ & $12.0(0.2)$ & $12.5(0.3)$ & $12.6(0.3)$ & \\
\hline$\beta \mathrm{H} / \beta \mathrm{S}$ & $1.40(0.04)$ & $1.23(0.04)$ & $1.19(0.05)$ & $1.23(0.02)$ & \\
\hline
\end{tabular}

$\alpha \mathrm{H}-\alpha$-helix, $\beta \mathrm{S}-\beta$-sheet, $\beta \mathrm{T}-\beta$-turns. Standard deviation in parentheses. 
into a more flexible form. Changes in the secondary structure of gluten samples in the presence of additives $(\mathrm{KCl}$ and cysteine) were studied by Mejri et al. (2005). Analysis of the amide I band showed an increase in the contribution of $\beta$-turns and $\beta$-sheets accompanied by a significant decrease in the $\alpha$-helix content. Thus, with the decreasing $\alpha$-helix contribution and the significant decrease in the value of the $\alpha \mathrm{H} / \beta \mathrm{S}$ ratio, gluten solubility the increased. Gluten solubility depended also on the $\beta$-turn content since these structures occurred most often at the protein surface due to their polar nature (Trevino et al., 2007). In the present studies, a decrease in the $\beta$-turn content was observed. Therefore, the solubility probably decreased due to inhibiting proteinwater interactions and worsening surface properties of the gluten (Chou and Fasman, 1997). Additionally, the observed shift of the amide I band maximum to shorter wavenumbers (Fig. 3a) indicated that the conformational changes were probably connected with cleavage of peptide bonds. Liao et al. (2010) studied the influence of the process of gluten deamination by succinic acid and citric acid on the secondary structure of the protein. Changes in the percentage contribution of structural components were observed but there was no maximum shift. Therefore, the conformational changes in gluten were mainly caused by increased electrostatic repulsion and a decrease in the number of hydrogen bonds (Liao et al., 2010).

The changes in the secondary structure of the treated gluten were significant, therefore, the factors causing them should be found. For this reason, wheat samples were treated by water and the aqueous solution of trisodium citrate. Figure 4 depicted the FTIR spectra (panel a) and their second derivatives (panel b) of gluten washed out from the wheat grain treated with water. The absorbance maximum of the amide I band was not shifted after using water and was located at $1648 \mathrm{~cm}^{-1}$. The zero shift of the amide I band maximum indicated that the secondary conformational changes were mainly caused by electrostatic interactions and a decrease in the number of hydrogen bonds (Liao et al., 2010). The percentage contribution of the structural components is shown in Table 1. Application of water caused significant changes in all the structural components in the gluten samples. On average, the $\alpha$-helix content decreased to $48.1 \%( \pm 0.4 \%)(\mathrm{p}<0.0001)$, while $\beta$-sheet structures and $\beta$-turns increased to $38.7 \%( \pm 0.4 \%)(\mathrm{p}<0.0001)$ and $13.2 \%$ $( \pm 0.5 \%)(p=0.0006)$, respectively, in comparison with the control sample. The value of the $\alpha \mathrm{H} / \beta \mathrm{S}$ ratio decreased to $1.24( \pm 0.02)$ after application of water.

Silver nanoparticles were reduced and stabilized by trisodium citrate, which can be regarded as a factor causing changes in the secondary structure of gluten proteins. For this reason, the wheat grains were treated with the aqueous solution of the compound at a fixed concentration. The FTIR spectra (panel a) and the second derivatives (panel b) of gluten samples washed out from the wheat grain treated with TC are depicted in Fig. 5. Table 1 presents the percentage

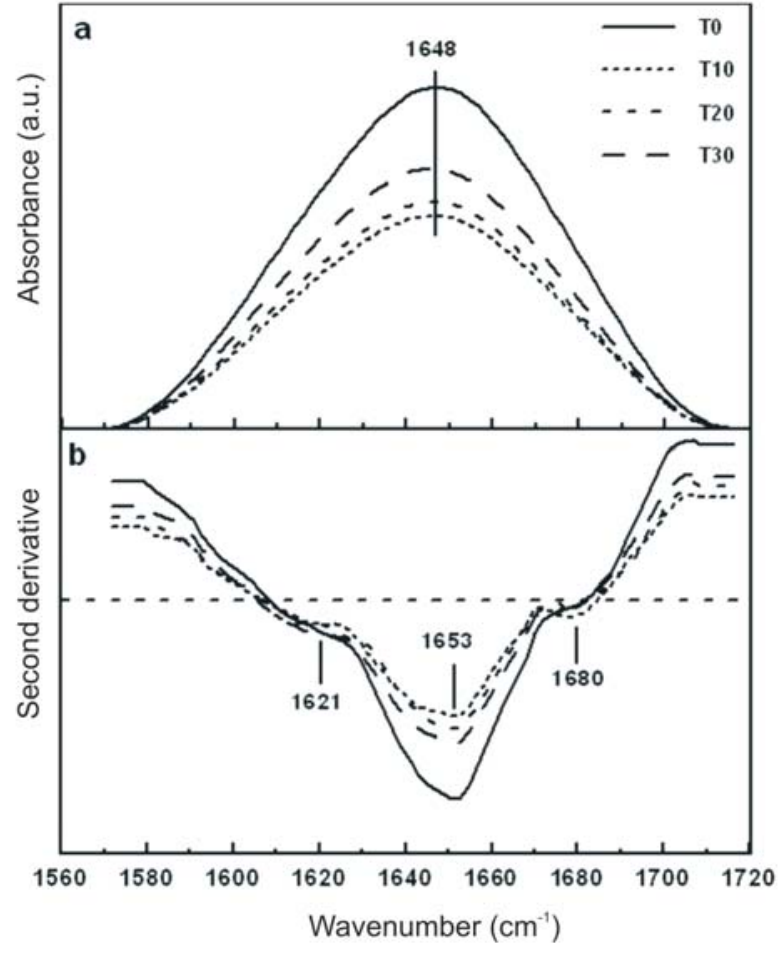

Fig. 4. Infrared spectra (a) and second derivatives (b) in the amide I region of untreated gluten (T0) and water-treated gluten (T10, T20, T30). The dashed line is placed at zero point.

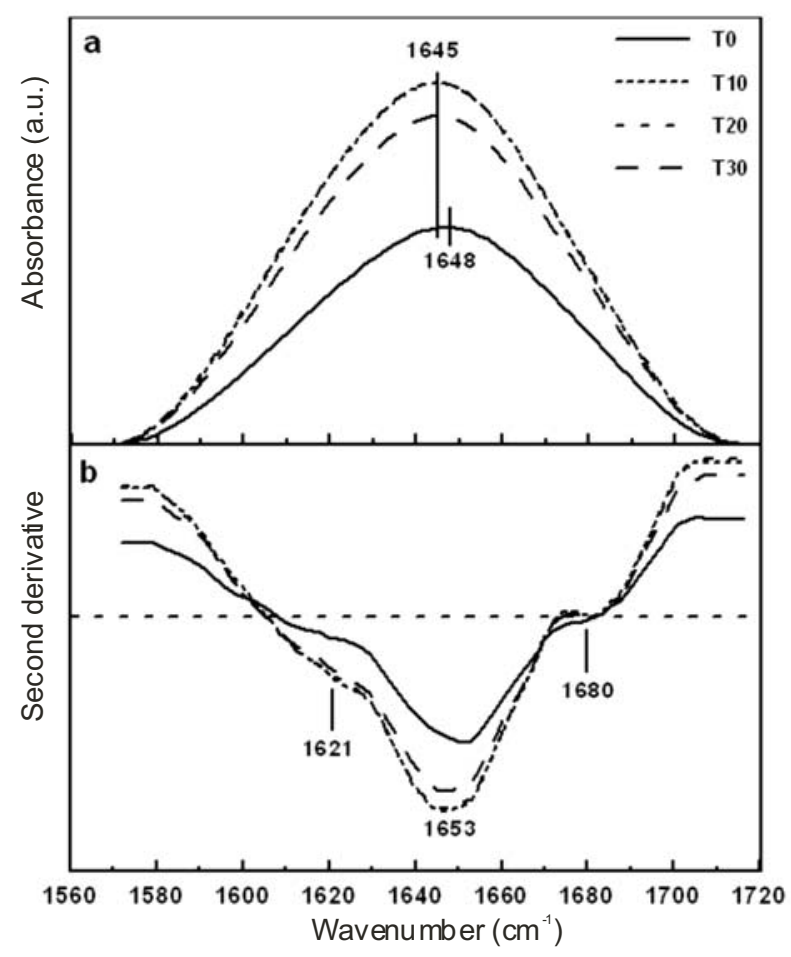

Fig. 5. Infrared spectra (a) and second derivatives (b) in the amide I region of untreated gluten (T0) and trisodium citrate-treated gluten (T10, T20, T30). The dashed line is placed at zero point. 

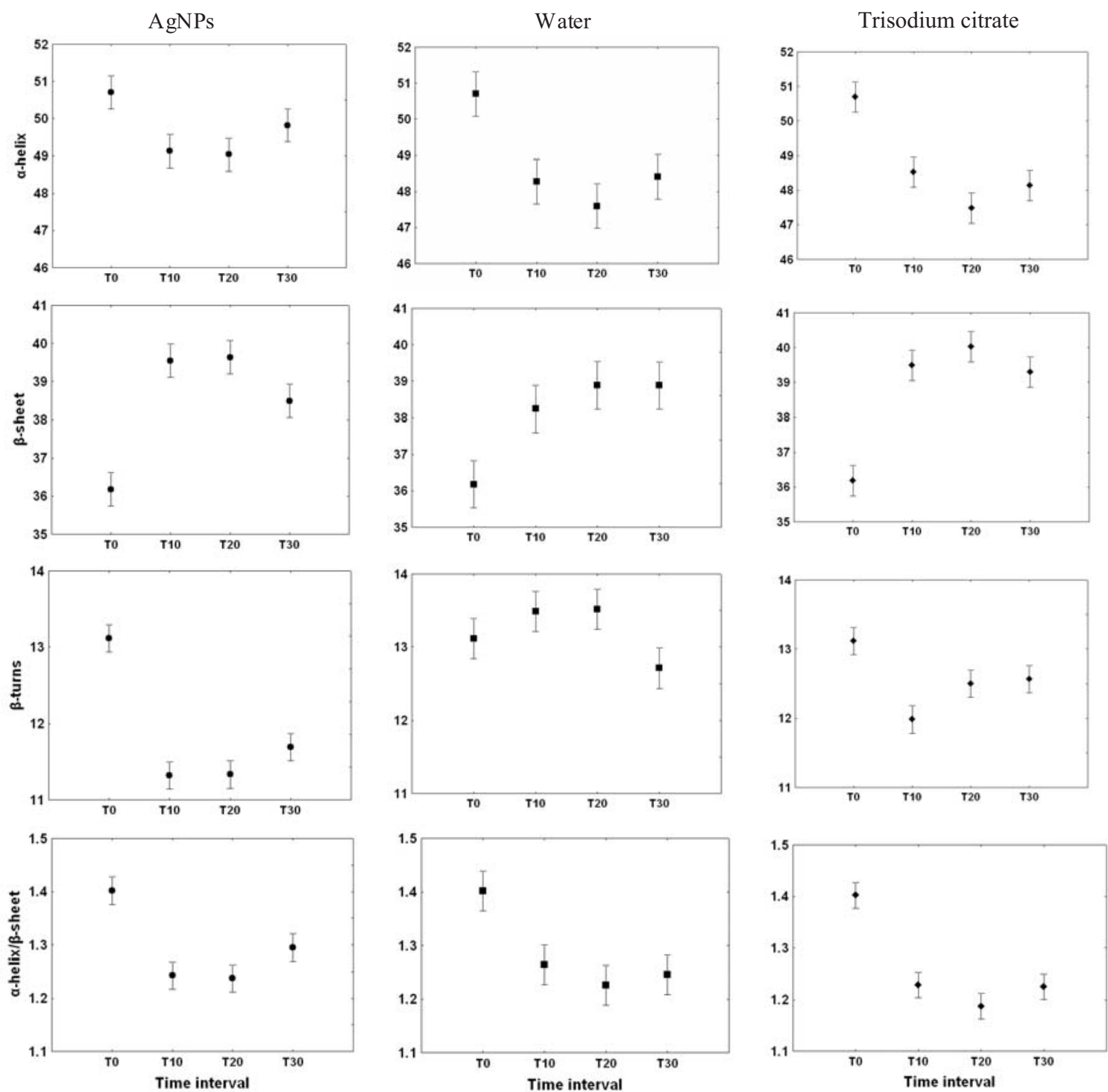

Fig. 6. Presentation of time-course changes in the secondary structure of wheat gluten after treating the wheat grain by the aqueous solution of silver nanoparticles and trisodium citrate and with water.

distribution of the structural components contained in gluten. Similar to the AgNP-treated samples, the FTIR spectra of the amide I band showed a slight shift of the absorption maximum from 1648 to $1645 \mathrm{~cm}^{-1}$. This shift can be connected with cleavage of peptide bonds (Liao et al., 2010). The TC-treated gluten samples were composed of approximately $48.0 \%( \pm 0.5 \%)$ of $\alpha$-helices, $39.6 \%( \pm 0.4 \%)$ of $\beta$-sheets, and $12.4 \%( \pm 0.3 \%)$ of $\beta$-turns. The ratio was equal to $1.22( \pm 0.02)$. All the parameters showed a significant correlation with the stirring time $(\mathrm{p}<0.0001)$.

Figure 6 presents changes in the secondary structure ( $\alpha \mathrm{H}, \beta \mathrm{S}, \beta \mathrm{T}$ content and $\alpha \mathrm{H} / \beta \mathrm{S}$ ratio) of gluten samples treated with the aqueous solution of silver nanoparticles and trisodium citrate and with water with respect to the stirring time (T0, T10, T20 and T30). Application of the chosen compounds revealed that the content of $\beta$-sheets increased at the expense of $\alpha$-helices. In the case of water, this increase was smaller in comparison with the remaining compounds. Therefore, a decrease in the value of the $\alpha$-helix-to- $\beta$-sheet ratio was observed. This decrease can be connected with the increase in gluten flexibility (Liao et al., 2009). Different changes were observed in the case of the $\beta$-turn content. The percentage distribution of this conformational structure diminished after using solutions of AgNPs and trisodium citrate, but increased after using water. The increase in the $\beta$-turn content indicated that protein-water interactions were facilitated as well as surface properties of gluten were improved (Chou and Fasman, 1997).

The results presented above indicated that changes in the secondary structure of the AgNP-treated gluten could be connected mainly with the influence of trisodium citrate. $\mathrm{TC}$ was used as both a reducer and stabilizer during the 
preparation of silver nanoparticles. A small number of TC molecules could not adsorb on the nanoparticle surface or dissociate from the surface and stay free in the solution. The aqueous solution of TC caused bigger changes in the $\alpha$-helix content $(48.0 \%)$ in comparison with AgNPs (49.3\%). The changes in the $\beta$-sheet content were comparable (39.2 and $39.6 \%$ for AgNPs and TC, respectively), whereas the content of $\beta$-turn was lower for AgNPs (11.4\%) than TC (12.4\%). As a result, the $\alpha \mathrm{H} / \beta \mathrm{S}$ ratio was smaller for the TC solution (1.22) in comparison with AgNPs (1.26). It can be concluded that free molecules of TC interact with gluten molecules causing an increase in gluten flexibility.

\section{CONCLUSIONS}

1. The changes in the gluten structure were connected mainly with the trisodium citrate action due to presence of a small number of free molecules of the stabilizer in the silver nanoparticle solution.

2 . The conformational changes in gluten pointed out that gluten flexibility increased (decrease in the $\alpha \mathrm{H} / \beta \mathrm{S}$ ratio from 1.40 for the control sample to 1.26 for the silver nanoparticle-treated samples) and the solubility of gluten decreased (a decrease in the $\beta$-turn content from 13.1 to $11.4 \%)$.

3. Generally, the colloidal solution of silver nanoparticles stabilized by trisodium citrate affected the secondary structure of wheat gluten proteins. An increase in the $\beta$-sheet content was observed at the expense of the $\alpha$-helix and $\beta$-turns content as well as a decrease in the value of the $\alpha \mathrm{H} / \beta \mathrm{S}$ ratio. These conformational changes could be connected with the cleavage of peptide bonds because the maximum of the amide I band was shifted to shorter wavenumbers.

\section{REFERENCES}

Ahamed M., AlSahli M.S., and Siddiqui M.K.J., 2010. Silver nanoparticles applications and human health. Clin. Chim. Acta, 411, 1841-1848.

American Association of Cereal Chemists International, 2000. Approved Methods of the AACC, Association, St. Paul, MN, USA.

Barrena R., Casals E., Colon J., Font, X., Sanchez A., and Puntes V., 2009. Evaluation of the ecotoxicity of model nanoparticles. Chemosphere, 75, 850-857.

Bouwmeester H., Dekkers S., Noordam M.Y., Hagens W.I., Bulder A.S., de Heer C., ten Voorde S.E.C.G., Wijnhoven S.W.P., Marvin H.J.P., and Sips A.J.A.M., 2009. Review of health safety aspects of nanotechnologies in food production. Regul. Toxicol. Pharm., 53, 52-62.
Bystrzejewska-Piotrowska G., Golimowski J., and Urban P.L., 2009. Nanoparticles: their potential toxicity, waste and environmental management. Waste Manag., 29, 2587-2595.

Cai S. and Singh B.R., 1990. Identification of $\beta$-turn and random coil amide III infrared bands for secondary structure estimation of proteins. Biophys. Chem., 80, 7-20.

Carbonaro M. and Nucara A., 2010. Secondary structure of food proteins by Fourier transform spectroscopy in the midinfrared region. Amino Acids, 38, 679-690.

Chou P.Y. and Fasman G.D., 1997. $\beta$-turns in proteins. J. Mol. Biol., 115, 135-175.

Duran N., Marcarto P.D., De Souza G.I.H., Alves O.L., and Esposito E., 2007. Antibacterial effect of silver nanoparticles produced by fungal process on textile fabrics and their effluent treatment. J. Biomed. Nanotechnol., 3, 203-208.

Fernandez A., Soriano E., Lopez-Carballo G., Picouet P., Lloret E., Gavara R., and Hernandez-Munoz P., 2009. Preservation of aseptic conditions in absorbent pads by using silver nanotechnology. Food Res. Int., 42, 1105-1112.

Guzman M.G., Dille J., and Godet S., 2009. Synthesis of silver nanoparticles by chemical reduction method and their antibacterial activity. Int. J. Chemical Biomolecular Eng., 2(3), 104-111.

Jayas D.S. and White N.D.G., 2003. Storage and drying of grain in Canada: Low cost approaches. Food Control, 14, 255-261.

Lee D.C., Haris P.I., Chapman D., and Mitchell R.C., 1990. Determination of protein secondary structure using factor analysis of infrared spectra. Biochemistry, 29, 9185-9193.

Lee P.C. and Meisel D., 1982. Adsorption and surface-enhanced Raman of dyes on silver and gold sols. J. Phys. Chem., 86, 3391-3395.

Liao L., Liu T.X., Zhao M.M., Ciu C., Yuan B.E., Tang S., and Yang F., 2010. Functional, nutritional and conformational changes from deamination of wheat gluten with succinic acid and citric acid. Food Chem., 123, 123-130.

Liao L., Zhao M., Zhao H., Ren J., Cui C., and Hu X., 2009. Effect of succinic acid deamination-induced modification on wheat gluten. Frontiers Chemical Eng. China, 3, 386-392.

Marambio-Jones C. and Hoek E.M.V., 2010. A review of the antibacterial effects of silver nanomaterials and potential implications for human health and the environment. J. Nanopart. Res., 12, 1531-1551.

Mejri M., Roge B., BenSouissi A., Michels F., and Mathhlouti M., 2005. Effects of some additives on wheat gluten solubility: A structural approach. Food Chem., 92, 7-15.

Nawrocka A. and Cieśla J., 2013. Influence of Ag-nanoaprticles on food components in wheat. Int. Agrophys., 27, 49-56.

Rai M., Yadav A., and Gade A., 2009. Silver nanoparticles as a new generation of antimicrobials. Biotechnol. Adv., 27, 76-8.

Surewicz W.K., Mantsch H.H., and Chapman D., 1993. Determination of protein secondary structure by Fourier transform infrared spectroscopy: a critical assessment. Biochemistry, 32, 389-394.

Trevino S.R., Schaefer S., Scholtz J.M., and Pace C.N., 2007. Increasing protein conformational stability by optimizing $\beta$-turn sequence. J. Mol. Biol., 373, 211-218. 